\title{
STUDY OF ARGON AFTERGLOW WITH THE AIR ADDITION
}

\author{
MazÁnková V. ${ }^{a, *}$, Trunec D. ${ }^{b}$, Petrová K. ${ }^{a}$, KrČMa F. ${ }^{a}$ \\ ${ }^{a}$ Faculty of Chemistry, Brno University of Technology, Purkyňova 118, 612 00 Brno, Czech Republic \\ ${ }^{b}$ Faculty of Science, Masaryk University, Kotlářská 2, 61137 Brno, Czech Republic \\ * mazankova@fch.vut.cz
}

\begin{abstract}
The reaction kinetics in argon flowing afterglow (post-discharge) with the air addition was studied by optical emission spectroscopy. The optical emission spectra were measured along the post-discharge flow tube. A zero-dimensional kinetic model for the reactions in the afterglow was developed. This model allows to calculate the time dependencies of particle concentrations.
\end{abstract}

Keywords: argon afterglow, optical emission spectroscopy, kinetic model.

\section{Introduction}

Besides different types of plasma argon plasma generated by electrical discharges has an exceptional position for its exploitation. A lot of treatment techniques and production of thin films and multilayer systems are arranged in argon plasma atmosphere [1. Special position has also argon afterglows containing different impurities. Afterglow systems containing oxygen or nitrogen have a wide range of applications in different fields due to the presence of reactive oxygen or nitrogen species (oxygen atoms, excited metastable oxygen and nitrogen molecules) [2, 3]. The afterglows of Ar$\mathrm{O}_{2}$ or Ar- $\mathrm{N}_{2}$ plasmas have been studied experimentally or theoretically by numerous groups. The published studies mainly aimed to determine the concentrations of reactive species in the treatment area. Theoretical kinetic model of Ar- $\mathrm{N}_{2}$ flowing afterglow was developed by Loiseau et al. [4] and recently a model for Ar- $\mathrm{N}_{2}$ mixture was also developed by Shneider et al. [5] in order to study population inversion in this gas mixture. A zero dimensional kinetic model in $\mathrm{Ar}-\mathrm{O}_{2}$ surface-wave microwave discharges was developed by Kutasi et al [6, 7] to investigate electron and heavy particle kinetics and dissociation of $\mathrm{O}_{2}$ molecules in the discharge and the flowing afterglow. The present work is focused on the experimental study of air addition directly to the argon flowing afterglow. The intensity of the arising nitrogen second positive system (SPS) was measured in dependence on decay time by optical emission spectroscopy. A simple kinetic model was developed in order to explain the experimental results.

\section{Experimental setup}

The flowing configuration of argon DC discharge with the air admixture was used for this experimental study. The experimental set-up was already used for our previous studies of argon afterglow with nitrogen admixture [8] and nitrogen afterglow with mercury vapour admixture [9]. A simplified schematic drawing of the experimental set-up is given in Figure 1. The active discharge was created in a quartz discharge tube with the inner diameter of $12 \mathrm{~mm}$ at the constant total gas pressure of $1000 \mathrm{~Pa}$ and the discharge power of $50 \mathrm{~W}$. Hollow molybdenum electrodes were placed in the side arms (at the interelectrode distance of $120 \mathrm{~mm}$ ) of the main discharge tube. The argon gas was of $99.99 \%$ purity and it was further cleaned by Oxiclear. The reactor system was pumped continuously by a rotary oil pump. The argon flow of $1400 \mathrm{sccm}$ was automatically controlled by the Bronkhorst mass flow controller. The synthetic air was prepared by mixing of nitrogen and oxgen $\left(8 \mathrm{sccm} \mathrm{N}_{2}+2 \mathrm{sccm}\right.$ $\mathrm{O}_{2}$ ) and flow was also automatically controlled by the Bronkhorst mass flow controllers. The moveable capillary tube for air addition was made of Pyrex and it was immersed upstream from the discharge into the quartz tube at its axis. Its external diameter was $2 \mathrm{~mm}$, the inner diameter was $0.5 \mathrm{~mm}$ and the length was $400 \mathrm{~mm}$. The position of the output end of capillary tube was fixed at $50 \mathrm{~mm}$ from the end of the active discharge (nearest edge of the side arm with cathode). The flow analysis was performed in the same way as in previous study on argon metastable quenching [8]. The input gas temperature was $300 \mathrm{~K}$.

The optical spectra were measured by Jobin Yvon monochromator TRIAX 550 with $300 \mathrm{gr} / \mathrm{mm}$ and $1200 \mathrm{gr} / \mathrm{mm}$ grating and with CCD detector. The emitted light was led to the entrance slit of the monochromator by the multimode quartz optical fibre movable along the discharge tube.

\section{Results and Discussion}

The optical emission spectra were measured in the active discharge with grating $1200 \mathrm{gr} / \mathrm{mm}$ and in the afterglow with grating $300 \mathrm{gr} / \mathrm{mm}$. The argon metastable concentration in the active discharge was determined from the spectra using the self-absorption method [10]. However, no self-absorption was observed, so the mean (across the tube diameter) metastable concentration was below $10^{9} \mathrm{~cm}^{-3}$. Only the SPS and argon lines were found in the spectra. The $\mathrm{N}_{2}(\mathrm{C})$ state, which is the upper state of SPS, is 


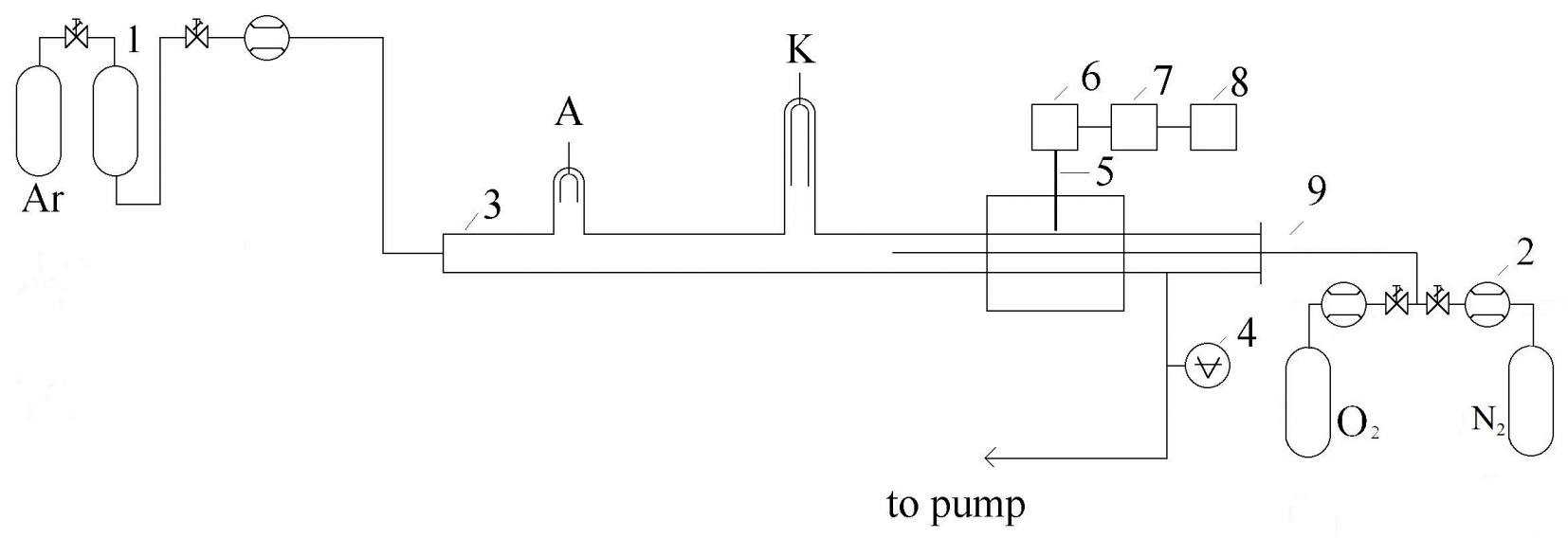

Figure 1. Scheme of the experimental setup. 1 - catalyzer Oxiclear; 2 - mass flow controllers; 3 - quartz discharge tube; 4 - capacitance gauge; 5 - quartz optical fibre; 6 - monochromator Jobin Yvon Triax 550; 7 - CCD; 8 PC; 9 - movable Pyrex capillary tube.

produced by excitation transfer in collisions of argon metastable $\operatorname{Ar}\left({ }^{3} \mathrm{P}_{2}\right)$ with nitrogen molecules.

As it can be seen from Figure 2, no transitions from the upper state with $v>2$ were observed, which is typical for the population of $\mathrm{N}_{2}(\mathrm{C})$ state by excitation transfer from $\operatorname{Ar}\left({ }^{3} \mathrm{P}_{2}\right)$ state.The SPS intensity was measured as a function of position along the flow tube. The SPS was identified 20 millimeters before the addition point. An example of the recorded post-discharge spectrum after air addition is shown in Figure 2 The dependence of SPS intensity on the distance from active discharge is shown in Figure 3 This dependence can be divided into two parts. The first part represents a rapid increase of SPS intensity to the maximal value due to the mixing of air with flowing argon.

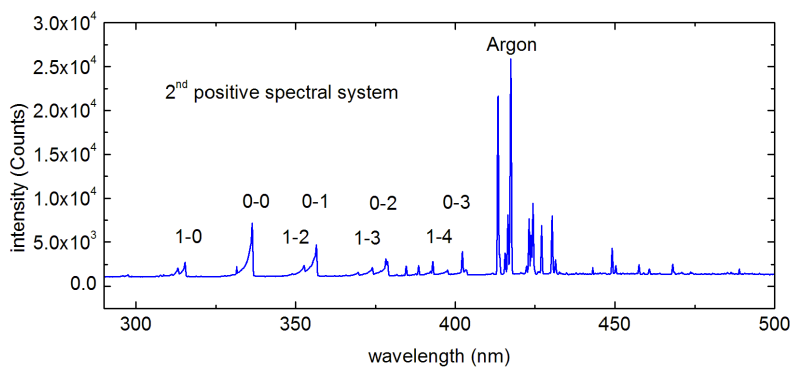

Figure 2. Overview spectrum of argon post-discharge with air titration.

In the second part the SPS intensity decreases due to the decrease of argon metastable concentration and thus due to the decrease of excitation transfer rate. A zero-dimensional kinetic model for the argon - air afterglow was developed. The initial concentrations of argon $[\mathrm{Ar}]_{0}$, molecular oxygen $\left[\mathrm{O}_{2}\right]_{0}$ and molecular nitrogen $\left[\mathrm{N}_{2}\right]_{0}$ in ground states were calculated from the equation of state for ideal gas for $T=300 \mathrm{~K}$ and from corresponding flow rates. The concentration of argon ground state was considered to be constant. At our experimental conditions the electrons are thermal-

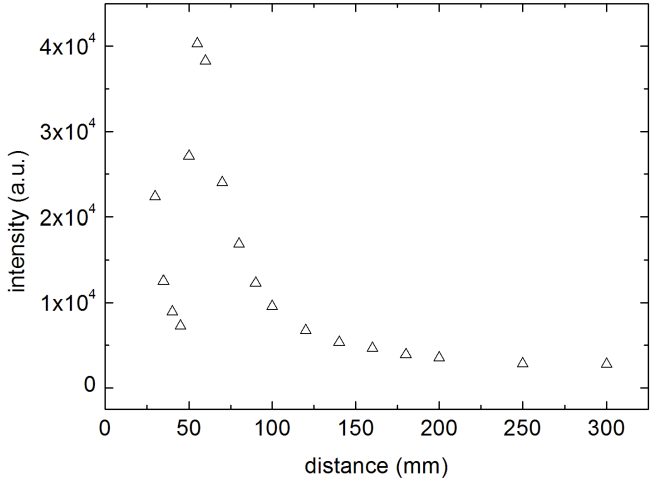

Figure 3. The dependence of SPS intensity (0-1 transition) on distance from active discharge.

ized due to collisions with argon atoms. The time needed for the electron temperature decrease within $10 \%$ of neutral gas temperature was calculated using data published by Trunec et al. 11. This calculated time for estimated initial electron energy of $4 \mathrm{eV}$ was $1.2 \mathrm{~ms}$, which corresponds to the distance of $2.4 \mathrm{~cm}$ in the flow tube. So, the electron temperature was equal to neutral gas temperature $(300 \mathrm{~K})$ in our experiment and this electron temperature was also used in the kinetic model. The reactions involved in the model and their rate coefficients were taken from [8, 9, 12]. The resulting system of differential equations was solved numerically. The results of the kinetic model are shown in Figures 4, 5 and 6.

The Figure 4 shows the time dependence of electron and ion concentrations. During the first hundred $\mu \mathrm{s}$, the $\mathrm{Ar}^{+}$ions are converted to $\mathrm{Ar}_{2}^{+}$molecular ions, however both these ions react with atomic and molecular oxygen and also with molecular nitrogen in charge transfer reactions producing $\mathrm{O}^{+}, \mathrm{O}_{2}^{+}$and $\mathrm{N}_{2}^{+}$ ions. Thereafter the $\mathrm{O}^{+}$ions and all nitrogen ions are 


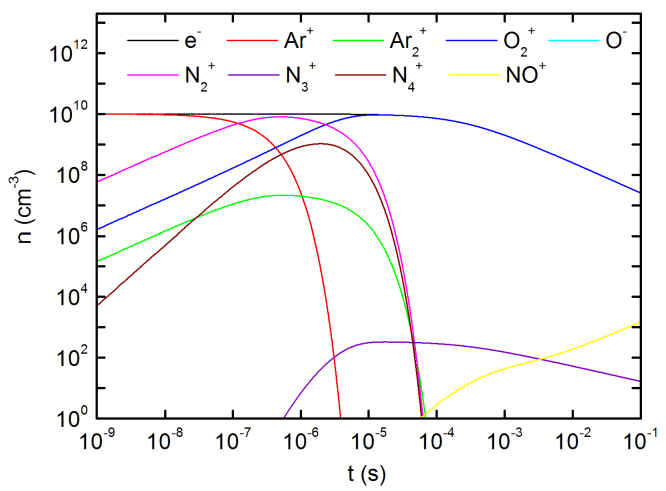

Figure 4. The calculated time dependence of electron and ion concentrations in the afterglow.

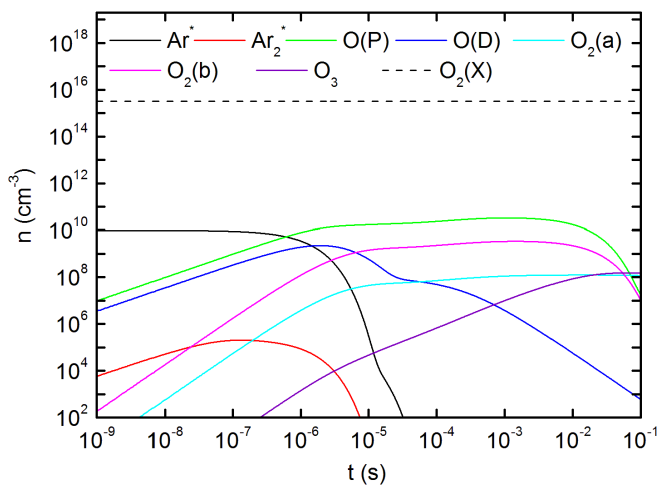

Figure 5. The calculated time dependence of neutral particle concentrations in the afterglow.

converted to $\mathrm{O}_{2}^{+}$ions by exothermic charge transfer reaction. So, after the first millisecond all positive ions are converted to $\mathrm{O}_{2}^{+}$ions. Thus, after the first millisecond only electrons and $\mathrm{O}_{2}^{+}$ions are the remaining charged particles, which recombine mutually and their concentrations further decrease and also the $\mathrm{O}_{2}^{+}$ ions react again in exothermic charge transfer reaction with arising NO.

The Figure 5 shows the time dependence of neutral oxygen species and excited argon and oxygen species concentrations. The $\mathrm{Ar}^{*}$ metastables produced in the discharge are converted to excimer $\mathrm{Ar}_{2}^{*}$ molecules, both $\mathrm{Ar}^{*}$ and $\mathrm{Ar}_{2}^{*}$ react with molecular oxygen producing atomic oxygen in ground or excited state. After the first millisecond, the concentrations of argon excited species are negligibly small. Mutual reactions of $\mathrm{O}$ and $\mathrm{O}_{2}$ produce ozone and metastable oxygen molecules $\mathrm{O}_{2}(\mathrm{a})$ and $\mathrm{O}_{2}(\mathrm{~b})$. So after the first millisecond the remaining neutral particles with significant concentrations in afterglow are atomic oxygen in ground state $\mathrm{O}(\mathrm{P})$, metastable molecular oxygen states $\mathrm{O}_{2}(\mathrm{a})$ and $\mathrm{O}_{2}(\mathrm{~b})$ and ozone together with argon and molecular oxygen in ground states. The concentration of metastable atomic oxygen $\mathrm{O}(\mathrm{D})$ is very low

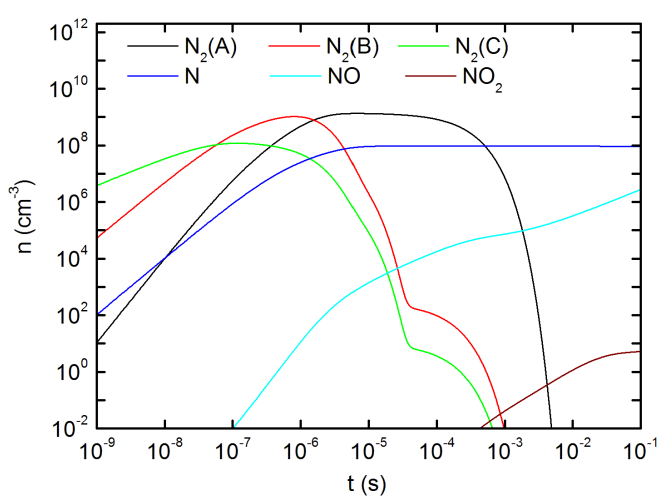

Figure 6. The calculated time dependence of nitrous oxides concentrations in the afterglow.

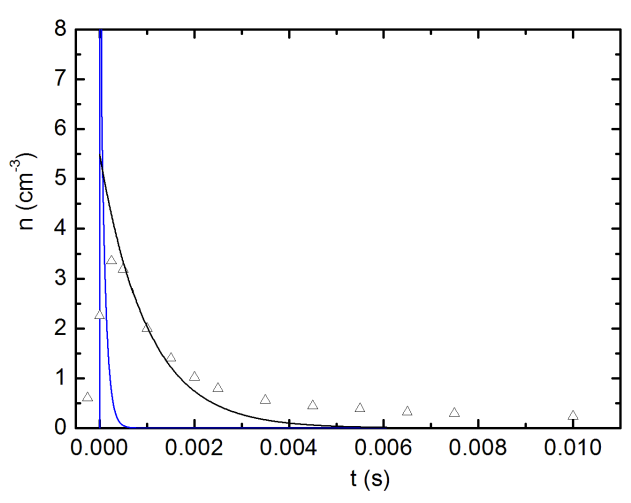

Figure 7. The comparison of calculated $N_{2}(C)$ concentration with measured SPS intensity. Blue linecalculated $N_{2}(C)$ concentration, black line - convolution of calculated $N_{2}(C)$ concentration with apparatus function, triangles - measured SPS intensities.

(around $10^{4} \mathrm{~cm}^{-3}$ ).

The Figure 6 shows the time dependence of nitrogen and nitrous oxides species concentrations. The $\mathrm{N}_{2}(\mathrm{C})$ states produced in excitation transfer reaction decays to $\mathrm{N}_{2}(\mathrm{~B})$ which decays further to $\mathrm{N}_{2}(\mathrm{~A})$. All these three nitrogen excited states are also quenched by $\mathrm{O}_{2}$ producing oxygen atoms. The nitrogen atoms are produced in collisions of two $\mathrm{N}_{2}(\mathrm{~A})$ molecules. The atomic oxygen and nitrogen then react and nitrous oxides are produced.

The results from the kinetic model are compared with measured SPS intensities in Figure 7

In order to compare directly the results of model with experimental results the convolution of kinetic model results with an apparatus function of experimental setup was calculated [8]. The apparatus function accounts for the light propagation in flow tube wall. This light propagation in the wall leads to the slower decrease of SPS intensity in the experiment. So calculated SPS intensity decay is in good agreement with experimental data except the times longer than $5 \mathrm{~ms}$, when there is still very low nonzero experimentally 
measured SPS intensity. The origin of this very low SPS intensity is not clear.

\section{Conclusions}

The flowing argon afterglow with air addition was studied using optical emission spectroscopy. The optical emission spectroscopy was used to measure the nitrogen SPS along the flow tube, which arises in excitation transfer between argon metastables and nitrogen molecules. The kinetic model shows time dependences of different species in the afterglow. The argon and nitrogen excited states decay very quickly mainly by quenching with molecular oxygen. Also the argon and nitrogen ions decay very quickly due to charge transfer reactions to molecular oxygen. So after first millisecond only oxygen species are important. Then atomic oxygen reacts with atomic nitrogen producing $\mathrm{NO}$ and further $\mathrm{NO}_{2}$.

\section{Acknowledgements}

The research was supported by Czech Ministry of Education, Youth and Sports, project No. COST CZ LD15010 and within the collaboration of the COST Action CM 1401.

\section{References}

[1] S. Saloum, M. Naddaf, and B. Alkhaled. Diagnostics of $\mathrm{N}_{2}$-Ar plasma mixture excited in a $13.56 \mathrm{MHz}$ hollow cathode discharge system: application to remote plasma treatment of polyamide surface. J. Phys. D: Appl. Phys., 41(4):045205, 2008.

[2] M. Mafra, T. Belmonte, F. Poncin-Epaillard, A. Maliska, and U. Cvelbar. Treatment of hexatriacontane by $\mathrm{Ar}-\mathrm{O}_{2}$ remote plasma: Formation of the active species. Plasma Process. Polym., 6(S):S198-S203, 2009.

[3] S. Lerouge, M. R. Wertheimer, and L'H. Yahia. Plasma sterilization: a review of parameters, mechanisms, and limitations. Plasma Polym., 6(3):175-188, 2001.

[4] J. F. Loiseau, P. Pignolet, and B. Held. Numerical simulation of Ar- $\mathrm{N}_{2}$ excitation transfer in flowing afterglow. J. Phys. D: Appl. Phys., 25(5):745-750, 1992.

[5] M. N. Shneider, A. Baltuška, and A. M. Zheltikov. Population inversion of molecular nitrogen in an Ar: $\mathrm{N}_{2}$ mixture by selective resonance-enhanced multiphoton ionization. J. Appl. Phys., 110(8):083112, 2011.

[6] K. Kutasi, P. Sá, and V. Guerra. $\mathrm{O}_{2}$ dissociation in Ar- $\mathrm{O}_{2}$ surface-wave microwave discharges. J. Phys. D: Appl. Phys., 45(19):195205, 2012.

[7] K. Kutasi, R. Zaplotnik, G. Primc, and M. Mozetic. Controlling the oxygen species density distributions in the flowing afterglow of $\mathrm{O}_{2} / \mathrm{Ar}-\mathrm{O}_{2}$ surface-wave microwave discharges. J. Phys. D: Appl. Phys., 47(2):025203, 2014.

[8] V. Mazánková, D. Trunec, and F. Krčma. Study of argon flowing afterglow with nitrogen injection. $J$. Chem. Phys., 139(3):164311, 2013.

[9] V. Mazánková, D. Trunec, and F. Krčma. Study of nitrogen flowing afterglow with mercury vapor injection. J. Chem. Phys., 141(3):154307, 2014.
[10] M. Schulze, A. Yanguas-Gil, A. von Keudell, and Awakowicz P. A robust method to measure metastable and resonant state densities from emission spectra in argon and argon-diluted low pressure plasmas. J. Phys. D: Appl. Phys., 41(3):065206, 2008.

[11] D. Trunec, P. Spanel, and D. Smith. The influence of electron-electron collisions on electron thermalization in He and Ar afterglow plasmas. Chem. Phys. Lett., 372:728-732, 2003

[12] C. D. Pintassilgo, J. Loureiro, G. Cernogora, and M. Touzeau. Methane decomposition and active nitrogen in a $\mathrm{N}_{2}-\mathrm{CH}_{4}$ glow discharge at low pressures. Plasma Sources Sci. Technol., 8:463-478, 1999. 